\title{
03
}

\section{Methods for modifications of harmonic spectra from laser-produced plasmas*}

\author{
(C) R.A. Ganeev
}

The Guo China-US Photonics Laboratory, Changchun Institute of Optics, Fine Mechanics and Physics, Changchun 130033, China

e-mail: rashid_ganeev@mail.ru

Received November 21, 2017

We review the studies of the modifications of harmonic spectra from laser-produced plasmas. The substantial spectral broadening and blue shift of the harmonics generating in the laser plasma during transformation of the spectrum of the laser radiation formed the filaments upon passage of femtosecond pulses through the argon gas is analyzed. We also discuss the studies of harmonic spectra variations that occur as a result of the passage of the laser pulses through the carbon- and metal-containing plasmas at the variable density of ablated species and intensity of driving pulse. To show other type of modulation of harmonic emission spectra, the plasmas containing the small molecules of silver produced during ablation of the bulk silver and silver nanoparticles at the conditions suitable for efficient harmonic generation of the ultrashort pulses propagating through the laser-produced plasmas were analyzed. The time-of-flight mass-spectroscopy studies of plasmas confirmed the presence of these species in plasmas.

DOI: $10.21883 /$ OS.2018.04.45751.270-17

* Полный текст статьи опубликован в английской версии журнала. 